\title{
U-shaped association between central body fat and the urinary albumin-to-creatinine ratio and microalbuminuria
}

Kathleen Dittmann ${ }^{* *}$, Anke Hannemann ${ }^{1}$, Henri Wallaschofski ${ }^{1}$, Rainer Rettig ${ }^{2}$, Sylvia Stracke ${ }^{3}$, Henry Völzke ${ }^{4}$, Matthias Nauck ${ }^{1}$ and Nele Friedrich ${ }^{1}$

\begin{abstract}
Background: The prevalence of obese and overweight patients has increased dramatically worldwide. Both are common risk factors for chronic kidney disease (CKD) as indicated by a diminished estimated glomerular filtration rate (eGFR) or microalbuminuria. This study aimed to investigate whether anthropometric parameters [waist circumference (WC), waist-to-height ratio (WHtR) and body mass index (BMI)] are associated with renal function in a population-based study of Caucasian subjects.

Methods: Data from 3749 subjects (1825 women) aged 20 to 81 years from the Study of Health in Pomerania (SHIP) were analysed. Renal indices, including the urinary albumin-to-creatinine ratio (UACR), microalbuminuria, eGFR and CKD, were studied. Parameters of anthropometry (WC, WHtR and BMI) were categorised into sex-specific quintiles.

Results: Analysis of variance (ANOVA) models, adjusting for age, sex, type 2 diabetes mellitus and hypertension, revealed that a high and low WC or WHtR and low BMI were independently related to a higher UACR. Logistic regression models confirmed these results with respect to UACR and showed that subjects with a high or low WC or a high WHtR had increased odds of microalbuminuria. The ANOVA models revealed no relations of the investigated anthropometric parameters with eGFR. However, subjects with high values for these parameters had increased odds of CKD.

Conclusions: Our results demonstrate U-shaped associations between markers of central fat distribution and UACR or microalbuminuria in the general population, suggesting that both obese and very thin subjects have a higher risk of renal impairment.
\end{abstract}

Keywords: Anthropometry, Chronic kidney disease, Estimated glomerular filtration rate, Microalbuminuria, Study of Health in Pomerania, Urinary albumin-to-creatinine ratio

\section{Background}

Chronic kidney disease (CKD) is a global public health problem with an increasing incidence and prevalence, poor outcomes and high cost [1]. The prevalence of recognized CKD among subjects in the U.S. national social insurance program Medicare increased three-fold between 2000 and 2009, from 2.7 to $8.5 \%$ [2]. In most patients, CKD is silent and commonly not detected until an advanced stage [3]. Being overweight [body mass

\footnotetext{
* Correspondence: kathleen.dittmann@uni-greifswald.de

'Institute of Clinical Chemistry and Laboratory Medicine, Ernst-Moritz-Arndt University of Greifswald, Ferdinand-Sauerbruch-Strasse NK, Greifswald D-17475, Germany

Full list of author information is available at the end of the article
}

index (BMI) $\left.25-30 \mathrm{~kg} / \mathrm{m}^{2}\right]$ and obesity (BMI $>30 \mathrm{~kg} / \mathrm{m}^{2}$ ) represent major risk factors for renal dysfunction $[4,5]$. Several studies [6-9] have shown that obesity is a risk factor for a diminished glomerular filtration rate (GFR) and CKD. Data from the Framingham Heart Study [7] revealed that $\mathrm{BMI}$ is a strong predictor for developing CKD. A retrospective case-control study [8] confirmed these findings and indicated that young overweight subjects had a three-fold higher risk of stage 5 CKD than lean control subjects.

Moreover, waist circumference (WC) and waist-to-hip ratio (WHR) as indices of visceral obesity have been reported to be even more sensitive predictors of late-stage renal disease than BMI [6]. Data from the Prevention of

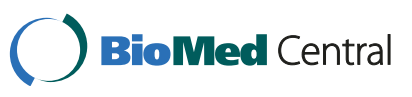


Renal and Vascular End-Stage Disease (PREVEND) study [9] revealed that obese subjects with a central fat distribution had a higher risk of microalbuminuria than lean subjects with a peripheral fat distribution. Furthermore, that study [9] showed that not only overweight and obese subjects but also lean subjects with central fat distribution were at a higher risk of a diminished GFR.

This study aimed to identify possible associations between WC, WHtR or BMI and renal dysfunction based on the urinary albumin-to-creatinine ratio (uACR), microalbuminuria, estimated GFR (eGFR) and CKD in an adult Caucasian population.

\section{Methods}

\section{Study population}

The Study of Health in Pomerania (SHIP) is a population-based cohort study in West Pomerania, a region in northeastern Germany. Details on the SHIP design, recruitment and procedures have been published elsewhere [10]. Baseline data collection started in October 1997 and was finished in March 2001. The initial sample for the baseline examination consisted of 4308 participants (net response: 69\%). All of the participants gave written informed consent. The study conformed to the principles of the Declaration of Helsinki as reflected by an a priori approval by the Ethics Committee of the University of Greifswald. Data usage for the project (number: SHIP/2011/50/D) was approved by the Research Network of Community Medicine and the manuscript was approved by the Publication Committee.

Of the 4308 participants, the 527 subjects with missing values for serum creatinine, urinary creatinine or serum albumin were excluded. Furthermore, 12 pregnant women and 20 subjects with missing values for anthropometric parameters or certain confounding factors were excluded. Altogether, the final study population included 3749 subjects (1924 men; 1825 women) aged 20 to 81 years.

\section{Data collection}

Information on age, sex, sociodemographic characteristics and medical history was collected using computerassisted personal interviews. The definition of type 2 diabetes mellitus was based on a self-reported physician diagnosis or use of an antidiabetic medication [anatomic, therapeutic and chemical (ATC) code: A10] during the last 7 days. After a five-minute resting period, systolic and diastolic blood pressures were measured three times on the right arm of seated subjects, using a digital blood pressure monitor (HEM-705CP, Omron Corporation, Tokyo, Japan). Each reading was followed by another rest period of three minutes. The mean of the second and third measurements was used for the statistical analyses. Hypertension was defined as systolic blood pressure $\geq 140 \mathrm{mmHg}$, diastolic blood pressure $\geq 90 \mathrm{mmHg}$ or self-reported use of antihypertensive medication.

Height was measured to the nearest $1 \mathrm{~cm}$ using a digital ultrasound instrument, and weight was measured to the nearest $0.1 \mathrm{~kg}$ in light clothing and without shoes using standard digital scales (Soehnle-Waagen $\mathrm{GmbH}$, Nassau, Germany). WC was measured to the nearest $0.1 \mathrm{~cm}$ using an inelastic tape midway between the lower rib margin and the iliac crest in the horizontal plane, with the subject standing comfortably with weight distributed evenly on both feet. The WHtR was calculated as the waist circumference in centimetres divided by the height in centimetres. The BMI was calculated as the weight in kilograms divided by the square of height in metres.

Non-fasting blood and urine samples were collected between 7.00 a.m. and 6.00 p.m. Blood samples were taken from the cubital vein of subjects in the supine position and prepared for immediate analysis or for storage at $-80^{\circ} \mathrm{C}$ for further analysis. Serum creatinine was measured using the Jaffé method (Hitachi 717; Roche Diagnostics, Mannheim, Germany). Urinary creatinine and albumin concentrations were determined using a Behring Nephelometer (Siemens BN albumin; Siemens Healthcare, Marburg, Germany) and a Hitachi 717 device (Roche Diagnostics), respectively. The uACR was calculated using the following equation: $\mathrm{uACR}(\mathrm{mg} / \mathrm{mmol})=$ urinary albumin concentration $(\mathrm{mg} / \mathrm{L}) /$ urinary creatinine concentration $(\mathrm{mmol} / \mathrm{L})$. Microalbuminuria was defined as an $\mathrm{uACR} \geq 2.5 \mathrm{mg} / \mathrm{mmol}$ in men or $\geq 3.5 \mathrm{mg} / \mathrm{mmol}$ in women [11].

The eGFR was calculated using the four-variable Modification of Diet in Renal Disease (MDRD) study equation: eGFR $=186.3 *$ serum creatinine $^{-1.154 *}$ age age $^{-0.203 *}$ (0.742 if female) [1,12]. CKD was defined as an eGFR $<60 \mathrm{~mL} / \mathrm{min} / 1.73 \mathrm{~m}^{2}$, consistent with the definition of CKD $\geq$ stage 3 proposed by the National Kidney Foundation Kidney Disease Outcomes Quality Initiative (KDOQI) [1].

\section{Statistical analyses}

Continuous data are expressed as the median (25th; 75th quartile) and nominal data are expressed as a percentage. For bivariate comparisons between women and men for the baseline characteristics of the study population, the Kruskal-Wallis test (continuous data) or the $\chi^{2}$-test (nominal data) was used. As a first step, linear regression with restricted cubic splines [13] was used to detect the possible dependency of eGFR or uACR on WC, WHtR or BMI. Three knots were prespecified, located at the $5^{\text {th }}, 50^{\text {th }}$ and $95^{\text {th }}$ percentile, as recommended by Stone and Koo [13], and the uACR values were log-transformed. Based on these results, the $\mathrm{WC}, \mathrm{WHtR}$ and $\mathrm{BMI}$ values were categorised into five 
groups according to sex-specific quintiles of distribution (Additional file 1: Table S1).

Next, analysis of variance (ANOVA) models were used to test for differences in the UACR or eGFR among sexspecific quintiles of WC, WHtR or BMI. Logistic regression models were performed to assess the associations between parameters of anthropometry and microalbuminuria or CKD. All models were adjusted for age, sex, type 2 diabetes mellitus and hypertension. Furthermore, we tested whether age or sex had a potential effect on our ordinal regression models, and no significant interaction $(\mathrm{p}<0.10)$ between age and the tested variable was found. Regarding the interaction between sex and the tested variable, only one out of six models detected a significant interaction (WHtR and microalbuminuria). Therefore, we decided not to perform age- or sexstratified analyses. Sensitivity analyses were performed after excluding subjects with type 2 diabetes mellitus or subjects who died within the first 5 years of follow up. Adjusted means and odds ratios (ORs) with 95\% confidence intervals (CIs) were calculated. A p-value $<0.05$ was considered to be statistically significant. Statistical analyses were performed using SAS 9.2 (SAS Institute Inc., Cary, NC).

\section{Results}

\section{General characteristics}

In our study population, the men were older and more often had hypertension than the women (Table 1). Regarding anthropometric parameters, the men were taller and had higher values for weight, WC, WHtR and BMI than the women. With respect to kidney function, the men more often had microalbuminuria and a higher eGFR, and less often had CKD, albeit they had higher serum creatinine concentrations than the women.

\section{Urinary albumin-to-creatinine ratio and estimated glomerular filtration rate}

First, linear regression models with restricted cubic splines revealed U-shaped associations between WC, WHtR or BMI and the uACR or eGFR (Figure 1, left panels). Multivariable ANOVA models confirmed these U-shaped relationships between WC or WHtR and uACR (Figure 1, upper right panel). Specifically, subjects in the lowest and highest WC and WHtR sex-specific quintiles had up to $0.29 \mathrm{mg} / \mathrm{mmol}$ higher uACRs than the subjects in the middle quintile. With respect to BMI, only subjects within the lowest group had higher uACRs than the remaining groups (uACRs in the lowest and highest BMI groups were $1.79 \mathrm{mg} / \mathrm{mmol}$ and $1.56 \mathrm{mg} /$ mmol, respectively). No associations were detected between the investigated anthropometric parameters and eGFR (Figure 1, lower right panel).

\section{Microalbuminuria and chronic kidney disease}

Logistic regression models were performed to assess the associations between anthropometric parameters and microalbuminuria or CKD. The analyses confirmed the U-shaped association between WC and microalbuminuria (Table 2). Subjects with a high or low WC had up to a $52 \%$ increased odds of microalbuminuria compared to the subjects in the middle quintile WC group. Furthermore, a high WHtR was related to increased odds of microalbuminuria, whereas the relationship between a low WHtR

Table 1 Characteristics of the study population

\begin{tabular}{|c|c|c|c|}
\hline Characteristics & Men $(n=1924)$ & Women $(n=1825)$ & p-value \\
\hline Age (years) & $52(37 ; 65)$ & $49(35 ; 62)$ & $<0.01$ \\
\hline Type 2 diabetes mellitus (\%) & 8.8 & 8.1 & 0.42 \\
\hline Hypertension (\%) & 62.9 & 41.8 & $<0.01$ \\
\hline Weight (kg) & $83.9(75.8 ; 92.7)$ & $68.8(60.9 ; 79.1)$ & $<0.01$ \\
\hline Height (cm) & $175(170 ; 180)$ & $163(158 ; 167)$ & $<0.01$ \\
\hline Waist circumference $(\mathrm{cm})$ & $95.5(87.5 ; 103.0)$ & $81.5(73.0 ; 92.1)$ & $<0.01$ \\
\hline $\mathrm{WHtR}$ & $0.55(0.50 ; 0.60)$ & $0.50(0.44 ; 0.57)$ & $<0.01$ \\
\hline $\mathrm{BMI}\left(\mathrm{kg} / \mathrm{m}^{2}\right)$ & $27.4(24.9 ; 30.0)$ & $26.2(22.8 ; 30.2)$ & $<0.01$ \\
\hline Serum creatinine (mg/dL) & $1.02(0.94 ; 1.11)$ & $0.87(0.80 ; 0.94)$ & $<0.01$ \\
\hline Urinary albumin (mg/L) & $8.5(4.8 ; 18.3)$ & $7.2(3.9 ; 14.5)$ & $<0.01$ \\
\hline Urinary creatinine $(\mathrm{mmol} / \mathrm{L})$ & $10.0(6.8 ; 13.8)$ & $6.7(4.1 ; 10.3)$ & $<0.01$ \\
\hline uACR (mg/mmol) & $0.83(0.51 ; 1.87)$ & $1.06(0.66 ; 2.00)$ & $<0.01$ \\
\hline Microalbuminuria (\%) & 19.3 & 14.5 & $<0.01$ \\
\hline eGFR $\left(\mathrm{mL} / \mathrm{min} / 1.73 \mathrm{~m}^{2}\right)$ & $83.24(73.92 ; 93.00)$ & $74.81(66.66 ; 84.46)$ & $<0.01$ \\
\hline CKD (\%) & 6.5 & 11.4 & $<0.01$ \\
\hline
\end{tabular}

$\mathrm{BMI}=$ body mass index; $\mathrm{CKD}=$ chronic kidney disease; eGFR = estimated glomerular filtration rate; $\mathrm{UACR}=$ urinary albumin-to-creatinine ratio; $\mathrm{WH} \mathrm{tR}=$ waist-to-height ratio. 

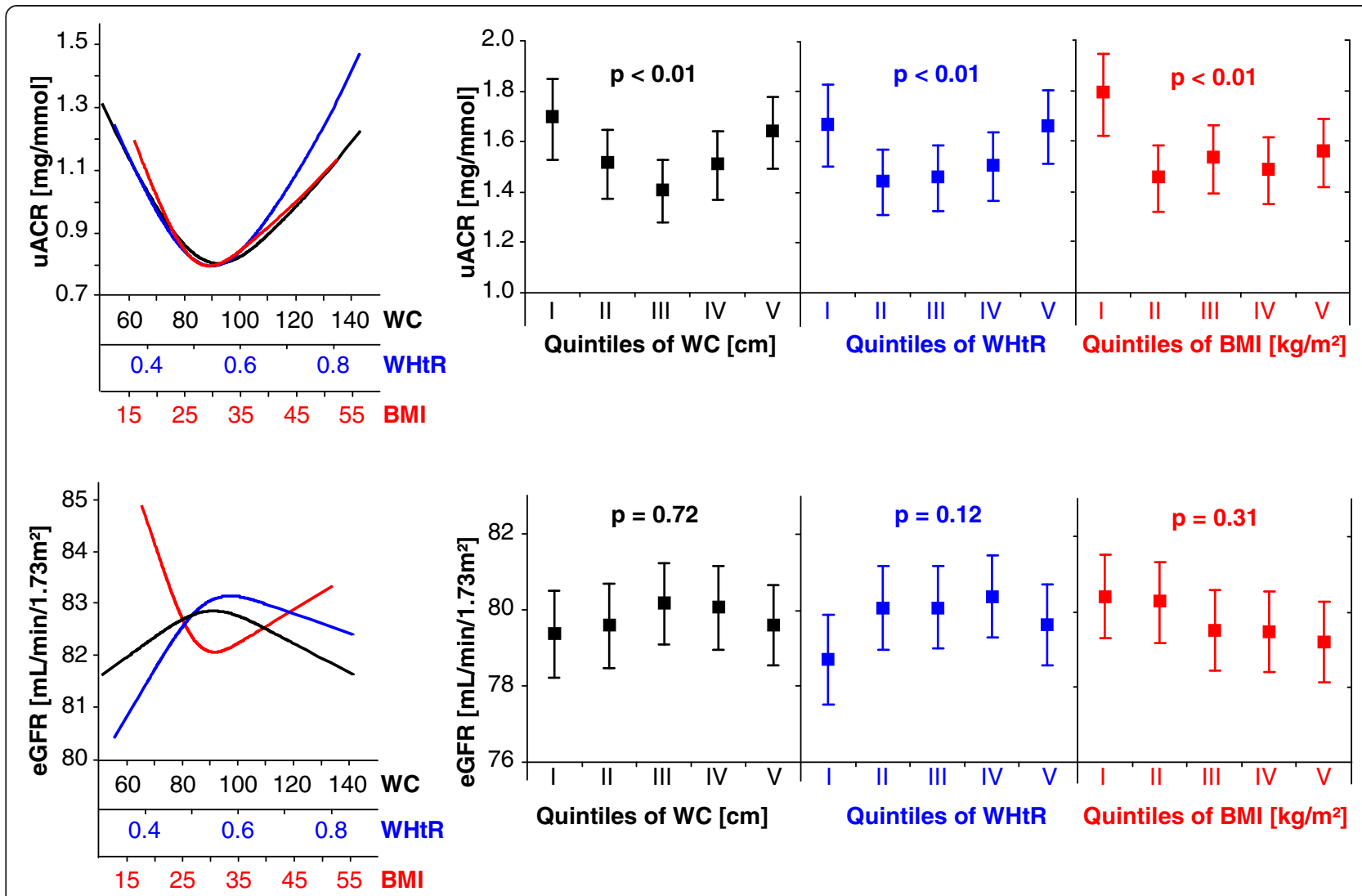

Figure 1 Association between waist circumference (WC), waist-to-height ratio (WHtR) or body mass index (BMI) and uACR (upper panels) or eGFR (lower panels) for the whole study population. Left side: Linear regression with restricted cubic splines. Right side: Adjusted mean uACR and adjusted mean eGFR with 95\% confidence intervals according to sex-specific quintiles of WC, WHtR or BMI calculated by analyses of variance. All models were adjusted for age, sex, type 2 diabetes mellitus and hypertension.

and microalbuminuria was not statistically significant. With respect to CKD, subjects in the highest quintiles for WC, WHtR or BMI exhibited up to $83 \%$ increased odds of CKD compared to subjects in the middle quintile group. No relationship was found between low anthropometric parameters and CKD.

Our main results were confirmed in several sensitivity analyses after excluding all subjects with type 2 diabetes mellitus (316 subjects), subjects who died within the first 5 years of follow-up, which indicates critical illness (259 subjects), or subjects with sample collection after noon (1037 subjects, Additional file 1: Table S2). Furthermore, HDL-cholesterol and total cholesterol were tested as further confounders (Additional file 1: Tables S3 and S4). All these analyses confirmed the main results and showed U-shaped associations between the considered anthropometric parameters and $\mathrm{UACR}$ as well as a positive relation between anthropometry and CKD.

\section{Discussion}

In this study, we observed associations between three different parameters of anthropometry and uACR or microalbuminuria. Both a low and high WC, WHtR and BMI positively correlated with the uACR. Furthermore, a high or low WC and a high WHtR were associated with increased odds of microalbuminuria. In contrast to the uACR, no association between any of the considered anthropometric parameters and eGFR was detected. However, subjects with high anthropometric values had higher odds of stage 3 CKD than subjects with average values.

In a previous study [14], obesity was related to an increased albumin excretion rate. Interestingly, in obese subjects with the same percentage of total body fat, the risk of abnormal albumin excretion was 18 times higher in those with central obesity but only four times higher in subjects with peripheral obesity compared to controls [14]. The PREVEND study [9] similarly showed that obese subjects with central fat distribution defined by an increased waist-to-hip ratio (WHR $\geq 0.9$ for men and $\geq 0.8$ for women) had an increased risk of microalbuminuria compared to obese subjects with a peripheral fat distribution (WHR $<0.9$ for men and $<0.8$ for women). Furthermore, not only obese but also lean or overweight subjects 
Table 2 Logistic regression models for the associations between sex-specific quintiles of WC, WHtR or BMI and microalbuminuria or CKD in the whole study population $(n=3749)$

\begin{tabular}{|c|c|c|c|c|}
\hline & \multicolumn{2}{|c|}{ Microalbuminuria } & \multicolumn{2}{|l|}{ CKD } \\
\hline & OR $(95 \% \mathrm{CI})$ & $p$-value & OR $(95 \% \mathrm{Cl})$ & $p$-value \\
\hline \multicolumn{5}{|c|}{ Waist circumference $(W C)[\mathrm{cm}]$} \\
\hline । & $1.48(1.06 ; 2.06)$ & 0.02 & $1.26(0.75 ; 2.11)$ & 0.38 \\
\hline$\|$ & $1.14(0.84 ; 1.55)$ & 0.40 & $1.12(0.73 ; 1.71)$ & 0.60 \\
\hline III & reference & & reference & \\
\hline IV & $1.21(0.91 ; 1.60)$ & 0.18 & $1.19(0.83 ; 1.71)$ & 0.35 \\
\hline V & $1.52(1.16 ; 2.00)$ & $<0.01$ & $1.63(1.15 ; 2.32)$ & $<0.01$ \\
\hline \multicolumn{5}{|c|}{ Waist-to-height ratio (WHtR) } \\
\hline । & $1.20(0.85 ; 1.71)$ & 0.30 & $1.56(0.87 ; 2.78)$ & 0.14 \\
\hline$\|$ & $0.91(0.66 ; 1.24)$ & 0.54 & $1.39(0.89 ; 2.17)$ & 0.14 \\
\hline III & reference & & reference & \\
\hline IV & $1.06(0.80 ; 1.39)$ & 0.69 & $1.18(0.81 ; 1.72)$ & 0.39 \\
\hline V & $1.33(1.03 ; 1.74)$ & 0.03 & $1.83(1.28 ; 2.60)$ & $<0.01$ \\
\hline \multicolumn{5}{|c|}{ Body mass index (BMI) $\left[\mathrm{kg} / \mathrm{m}^{2}\right]$} \\
\hline । & $1.28(0.94 ; 1.75)$ & 0.11 & $0.92(0.57 ; 1.48)$ & 0.72 \\
\hline$\|$ & $0.91(0.68 ; 1.23)$ & 0.56 & $0.86(0.56 ; 1.31)$ & 0.48 \\
\hline III & reference & & reference & \\
\hline IV & $1.01(0.76 ; 1.33)$ & 0.96 & $1.00(0.70 ; 1.44)$ & 0.99 \\
\hline V & $1.16(0.89 ; 1.53)$ & 0.27 & $1.47(1.04 ; 2.10)$ & 0.03 \\
\hline
\end{tabular}

$\mathrm{Cl}=$ confidence interval; $\mathrm{CKD}=$ chronic kidney disease; $\mathrm{OR}=$ odds ratio.

with a central fat distribution were at a higher risk of a diminished GFR than subjects with a peripheral fat distribution [9].

We also observed positive associations between high $\mathrm{WC}, \mathrm{WHtR}$ or BMI and the risk of CKD. These findings confirm the results of a community-based, cross-sectional study [15] that found a positive relationship between WHtR and the risk of CKD. There was no association between BMI and CKD in this study [15]. These results support the conclusion that WC [6] and WHtR [15] are more sensitive markers of CKD than BMI.

A study in 143 children with CKD [16] revealed that advanced CKD was associated with a low lean leg mass, indicating skeletal muscle wasting. Recent literature [17] suggests that muscle wasting in the context of CKD may be due to inflammation. Another study [18] showed that patients with a relatively modest degree of CKD are characterised by reduced lean body mass and bone mineral content. These findings reveal the complex relationships between anthropometry, metabolism and renal function and indicate that fat tissue measurement may contribute to the detection of renal impairment.

Central obesity is frequently associated with risk factors of CKD, such as hypertension $[19,20]$ and type 2 diabetes mellitus [20,21]. The PREVEND study [9] described higher systolic and diastolic blood pressures in overweight and obese subjects with a central fat distribution than in subjects with a peripheral fat distribution. During the last decades, adipose tissue has emerged as an active endocrine organ that releases several bioactive mediators, modulating haemostasis, blood pressure, lipid and glucose metabolism, inflammation and atherosclerosis [21]. Therefore, our findings of an association between WHtR or WC, as indices of central obesity [22], and the risk of microalbuminuria argue for an important role for visceral vs. peripheral fat in relation to albumin excretion.

Previous studies suggest that abdominal obesity may independently associate with microalbuminuria [9,14,23-27] . Data from the MONICA Augsburg survey 1994/95 [23] showed that microalbuminuric subjects had a higher WC, WHR and prevalence of elevated central adiposity and obesity than subjects without microalbuminuria. Another study [28] using measurements of visceral adipose tissue (VAT) and subcutaneous adipose tissue (SAT) revealed that VAT was associated with microalbuminuria in men but not in women and showed that microalbuminuria may represent a manifestation of visceral adiposity. However, our observation of an association between a low WC and risk of microalbuminuria argues against that assumption. Reason for these discrepancies might be the different definitions of obesity as well as microalbuminuria. Obesity might be based on WC, WHR, WHtR or BMI, which all do not differentiate between VAT and SAT accumulation. Furthermore, altered techniques used for the detection of microalbuminuria resulting in a reported prevalence of microalbuminuria ranging from $10 \%$ to $40 \%[29,30]$.

The pathophysiology underlying the association between low anthropometry and microalbuminuria is yet unknown. Which is partly part due to the fact that the pathophysiology leads to the development of microalbuminuria are not fully understood. The most likely reason is an alteration of intrarenal hemodynamics and may represent in hypertension as well as type 2 diabetes mellitus as an early unspecific feature of renal impairment. Even if the relation between low anthropometry and microalbuminuria is not fully explained, it is widely accepted that the association between BMI and all-cause mortality was U-shaped with the lowest mortality between $22.5-25 \mathrm{~kg} / \mathrm{m}^{2}$ [31]. Thus a low or high BMI is related to higher mortality risk. Furthermore, there is no doubt that microalbuminuria is a valid biomarker of increased mortality risk [32]. Therefore, microalbuminuria in low values of anthropometry might reflect a damage of glomerulus with complete different pathophysiology compared to vascular risk burden, like hypertension or diabetes, in obese people. There is good evidence of epidemiological studies that lowering fetal growth or low birth weight, result in an increased incidence of hypertension or renal impairment when the 
offspring reaches adulthood. One possible hypothesis might be, that conditions affecting fetal development leading to low birth weight are associated with renal programming or subclinical renal disease [33]. Microalbuminuria in such people might be one biomarker of these conditions, which is absolutely speculative at the moment.

In general, cross sectional epidemiological studies are not suited to estimate pathophysiology and causality. Therefore, further science, especially animals studies or interventional trials are needed to investigate the phenomenon of $\mathrm{U}$-shaped association between microalbuminuria and anthropometry in more detail.

Two limitations of our study merit special consideration. First, due to the lack of longitudinal data, we cannot detect causality between the anthropometric measures and renal dysfunction. Second, instead of complex and cost-intensive measurements of fat distribution, we used common measurements, such as height, weight and WC to assess the relationship between anthropometric parameters with renal dysfunction. These parameters are easy to measure in clinical practice and public health settings but may not be as accurate as measuring body fat distribution.

\section{Conclusions}

Our data suggest that both a high and low WC or WHtR and low BMI are associated with a higher uACR. Not only subjects with a high WC or WHtR but also subjects with a low WC may have an increased risk of microalbuminuria. However, our data provide no evidence that any of the considered anthropometric parameters are associated with eGFR. Nevertheless, subjects with a high WC, WHtR or BMI exhibit an increased risk of CKD compared to subjects with average anthropometric values.

\section{Additional file}

Additional file 1: Table S1. Percentiles of WC, WHtR and BMI in men
$(\mathrm{n}=1924)$ and women $(\mathrm{n}=1825)$. Table S2. Logistic regression models for
the associations between sex-specific quintiles of WC, WHtR or BMI and
microalbuminuria or CKD in the subjects time of examination before
noon ( $n=2712)$. Table S3. Logistic regression models for the
associations between sex-specific quintiles of WC, WHtR or BMl and
microalbuminuria (additionally adjusted for HDL-cholesterol and total
cholesterol) in the whole study population ( $\mathrm{n}=3749$ ). Table S4. Logistic
regression models for the associations between sex-specific quintiles of
WC, WHtR or BMl and CKD (additionally adjusted for HDL-cholesterol and
total cholesterol) in the whole study population ( $\mathrm{n}=3749$ ).

\section{Competing interests}

The authors declare that they have no competing interests.

\section{Authors' contributions}

Conception and Design: KD, AH, HW, NF. Data Analysis: KD. Interpretation of data: All. Article drafting: KD, NF, HW. Final approval: All authors read and approved the final manuscript.

\section{Acknowledgment}

This work is part of the research project Greifswald Approach to Individualized Medicine (GANI_MED). The GANI_MED consortium is funded by the BMBF and the Ministry of Cultural Affairs of the Federal State of Mecklenburg-West Pomerania (03IS2061A) and the Ministry of Cultural Affairs of the Federal State of Mecklenburg - West Pomerania (grant 03IS2061A). SHIP is part of the Community Medicine Research net of the University of Greifswald, Germany, which is funded by the Federal Ministry of Education and Research (grants no. 01ZZ9603, 01 ZZ0103 and 01ZZ0403), the Ministry of Cultural Affairs as well as the Social Ministry of the Federal State of Mecklenburg - West Pomerania. This study was also carried out in collaboration with the German Centre for Cardiovascular Research (GCCR) which is funded by the Federal Ministry of Education and Research and the Ministry of Cultural Affairs of the Federal State of Mecklenburg, West Pomerania.

\section{Author details}

${ }^{1}$ Institute of Clinical Chemistry and Laboratory Medicine, Ernst-Moritz-Arndt University of Greifswald, Ferdinand-Sauerbruch-Strasse NK, Greifswald D-17475, Germany. Institute of Physiology, Ernst-Moritz-Arndt University of Greifswald, Greifswald, Germany. ${ }^{3}$ Department of Internal Medicine A, Ernst-Moritz-Arndt University of Greifswald, Greifswald, Germany. ${ }^{4}$ Institute for Community Medicine, Ernst-Moritz-Arndt University of Greifswald, Greifswald, Germany.

Received: 18 June 2012 Accepted: 9 April 2013

Published: 17 April 2013

\section{References}

1. Levey AS, Coresh J, Balk E, Kausz AT, Levin A, Steffes MW, Hogg RJ, Perrone RD, Lau J, Eknoyan G: National Kidney Foundation practice guidelines for chronic kidney disease: evaluation, classification, and stratification. Ann Intern Med 2003, 139:137-147.

2. USRDS: Identification and care of patients with chronic kidney disease. 2011:45-58. http://www.usrds.org/2012/pdf/v1_ch2_12.pdf.

3. Whaley-Connell AT, Sowers JR, Stevens LA, MCFarlane SI, Shlipak MG, Norris KC, Chen SC, Qiu Y, Wang C, Li S, et al: CKD in the United States: Kidney Early Evaluation Program (KEEP) and National Health and Nutrition Examination Survey (NHANES) 1999-2004. Am J Kidney Dis 2008, 51:S13-20.

4. Hsu CY, McCulloch CE, Iribarren C, Darbinian J, Go AS: Body mass index and risk for end-stage renal disease. Ann Intern Med 2006, 144:21-28.

5. Foster MC, Hwang SJ, Larson MG, Lichtman JH, Parikh NI, Vasan RS, Levy D, Fox CS: Overweight, obesity, and the development of stage 3 CKD: the Framingham Heart Study. Am J Kidney Dis 2008, 52:39-48.

6. Ritz E: Metabolic syndrome and kidney disease. Blood Purif 2008, 26:59-62.

7. Fox CS, Larson MG, Leip EP, Culleton B, Wilson PW, Levy D: Predictors of new-onset kidney disease in a community-based population. JAMA 2004, 291:844-850.

8. Ejerblad E, Fored CM, Lindblad P, Fryzek J, McLaughlin JK, Nyren O: Obesity and risk for chronic renal failure. J Am Soc Nephrol 2006, 17:1695-1702.

9. Pinto-Sietsma SJ, Navis G, Janssen WM, de Zeeuw D, Gans RO, de Jong PE: A central body fat distribution is related to renal function impairment, even in lean subjects. Am J Kidney Dis 2003, 41:733-741.

10. Volzke H, Alte D, Schmidt CO, Radke D, Lorbeer R, Friedrich N, Aumann N, Lau K, Piontek M, Born G, et al: Cohort profile: the study of health in pomerania. Int J Epidemiol 2011, 40:294-307.

11. de Jong PE, Curhan GC: Screening, monitoring, and treatment of albuminuria: Public health perspectives. J Am Soc Nephrol 2006, 17:2120-2126.

12. Levey AS, Bosch JP, Lewis JB, Greene T, Rogers N, Roth D: A more accurate method to estimate glomerular filtration rate from serum creatinine: a new prediction equation. Modification of Diet in Renal Disease Study Group. Ann Intern Med 1999, 130:461-470.

13. Stone CKC: Additive splines in statistics. In Proceedings of the Statistical Computing Section ASA. Washington, DC: American Statistical Association; 1986:45-48.

14. Mulyadi L, Stevens C, Munro S, Lingard J, Bermingham M: Body fat distribution and total body fat as risk factors for microalbuminuria in the obese. Ann Nutr Metab 2001, 45:67-71.

15. Lin CH, Chou CY, Lin CC, Huang CC, Liu CS, Lai SW: Waist-to-height ratio is the best index of obesity in association with chronic kidney disease. Nutrition 2007, 23:788-793. 
16. Foster BJ, Kalkwarf HJ, Shults J, Zemel BS, Wetzsteon RJ, Thayu M, Foerster DL, Leonard MB: Association of chronic kidney disease with muscle deficits in children. J Am Soc Nephrol 2011, 22:377-386.

17. Mak RH, Cheung W: Cachexia in chronic kidney disease: role of inflammation and neuropeptide signaling. Curr Opin Nephrol Hypertens 2007, 16:27-31.

18. O'Sullivan AJ, Lawson JA, Chan M, Kelly JJ: Body composition and energy metabolism in chronic renal insufficiency. Am J Kidney Dis 2002, 39:369-375.

19. Hamm LL, Chen J: Fat chance for hypertension and chronic kidney disease. Hypertension 2011, 58:756-757.

20. Bombelli M, Facchetti R, Sega R, Carugo S, Fodri D, Brambilla G, Giannattasio C, Grassi G, Mancia G: Impact of body mass index and waist circumference on the long-term risk of diabetes mellitus, hypertension, and cardiac organ damage. Hypertension 2011, 58:1029-1035.

21. Rabe K, Lehrke M, Parhofer KG, Broedl UC: Adipokines and insulin resistance. Mol Med 2008, 14:741-751.

22. Gill T, Chittleborough C, Taylor A, Ruffin R, Wilson D, Phillips P: Body mass index, waist hip ratio, and waist circumference: which measure to classify obesity? Soz Praventivmed 2003, 48:191-200.

23. Liese AD, Hense HW, Doring A, Stieber J, Keil U: Microalbuminuria, central adiposity and hypertension in the non-diabetic urban population of the MONICA Augsburg survey 1994/95. J Hum Hypertens 2001, 15:799-804.

24. de Boer IH, Sibley SD, Kestenbaum B, Sampson JN, Young B, Cleary PA, Steffes MW, Weiss NS, Brunzell JD: Central obesity, incident microalbuminuria, and change in creatinine clearance in the epidemiology of diabetes interventions and complications study. J Am Soc Nephrol 2007, 18:235-243.

25. Chandie Shaw PK, Berger SP, Mallat M, Frolich M, Dekker FW, Rabelink TJ: Central obesity is an independent risk factor for albuminuria in nondiabetic South Asian subjects. Diabetes Care 2007, 30:1840-1844.

26. Lin CC, Liu CS, Li TC, Chen CC, Li Cl, Lin WY: Microalbuminuria and the metabolic syndrome and its components in the Chinese population. Eur J Clin Invest 2007, 37:783-790.

27. Kramer H, Reboussin D, Bertoni AG, Marcovina S, Lipkin E, Greenway FL 3rd, Brancati FL: Obesity and albuminuria among adults with type 2 diabetes: the Look AHEAD (Action for Health in Diabetes) Study. Diabetes Care 2009, 32:851-853.

28. Meredith C, Foster BA, LM G, Hwang SJ, Massaro JM, Hoffmann U, DeBoer IH, Robins SJ, Vasan RS, Fox CS: Association of subcutaneous and visceral adiposity with albuminuria: the Framingham Heart Study. Obesity (Silver Spring) 2011, 19:1284-1289.

29. Yudkin JS, Forrest RD, Jackson CA: Microalbuminuria as predictor of vascular disease in non-diabetic subjects. Islington Diabetes Survey. Lancet 1988, 2:530-533.

30. Damsgaard EM, Froland A, Jorgensen OD, Mogensen CE: Microalbuminuria as predictor of increased mortality in elderly people. BMJ 1990, 300:297-300.

31. Whitlock G, Lewington S, Sherliker P, Clarke R, Emberson J, Halsey J, Qizilbash N, Collins R, Peto R: Body-mass index and cause-specific mortality in 900000 adults: collaborative analyses of 57 prospective studies. Lancet 2009, 373:1083-1096.

32. Romundstad S, Holmen J, Kvenild $\mathrm{K}$, Hallan $\mathrm{H}$, Ellekjaer H: Microalbuminuria and all-cause mortality in 2,089 apparently healthy individuals: a 4.4-year follow-up study. The Nord-Trondelag Health Study (HUNT), Norway. Am J Kidney Dis 2003, 42:466-473.

33. Baum M: Role of the kidney in the prenatal and early postnatal programming of hypertension. Am J Physiol Renal Physiol 2010, 298:F235-247.

doi:10.1186/1471-2369-14-87

Cite this article as: Dittmann et al:: U-shaped association between central body fat and the urinary albumin-to-creatinine ratio and microalbuminuria. BMC Nephrology 2013 14:87.

\section{Submit your next manuscript to BioMed Central and take full advantage of:}

- Convenient online submission

- Thorough peer review

- No space constraints or color figure charges

- Immediate publication on acceptance

- Inclusion in PubMed, CAS, Scopus and Google Scholar

- Research which is freely available for redistribution

Submit your manuscript at www.biomedcentral.com/submit
C Biomed Central 